\title{
Anterior wall myocardial infarction in a young man caused by spontaneous dissection and hematoma of coronary artery
}

\author{
TOMASZ BOCHENEK* ${ }^{*}$ MICHAŁ LELEK, KATARZYNA MIZIA-STEC
}

\author{
Department of Cardiology, Medical University of Silesia, Katowice, Poland \\ *Corresponding author: Tomasz Bochenek; Department of Cardiology, Medical \\ University of Silesia, Ziołowa 47 street, Katowice 40-635, Poland; Phone: +48 3235980 00; Fax: +48 3225236 58; E-mail: tbochunl@gmail.com
}

(Received: January 31, 2018; Revised manuscript received: March 20, 2018; Second revised manuscript received: April 18, 2018;

Accepted: May 14, 2018)

Abstract: A 55-year-old man without any cardiac history has been admitted to Ist Department of Cardiology due to anterior wall infarction. In echocardiography (ECG), local anterior wall dysfunction has been observed, with good left ventricle ejection fraction. In angiography performed immediately after transfer to hospital, long lesion in left anterior descending coronary artery has been visualized with high angiographic suspicion of dissection and intramural coronary hematoma. Intravascular ultrasound (IVUS) has been performed and further confirmed the diagnosis of hematoma - LAD was stented using three coronary stents. IVUS has confirmed good position of stents. Integrillin has been used. Periprocedural time was uncomplicated. ECG showed resolution of myocardial infarction pattern and evolution of infarction has been observed. The patient was discharged home in good clinical condition. Coronary dissection and coronary hematoma are the potential cause of infarction and IVUS, despite optical coherence tomography being reference nowadays, is still a very valuable tool in diagnosis and treatment guiding in such cases.

Keywords: IVUS, angiography, infarction

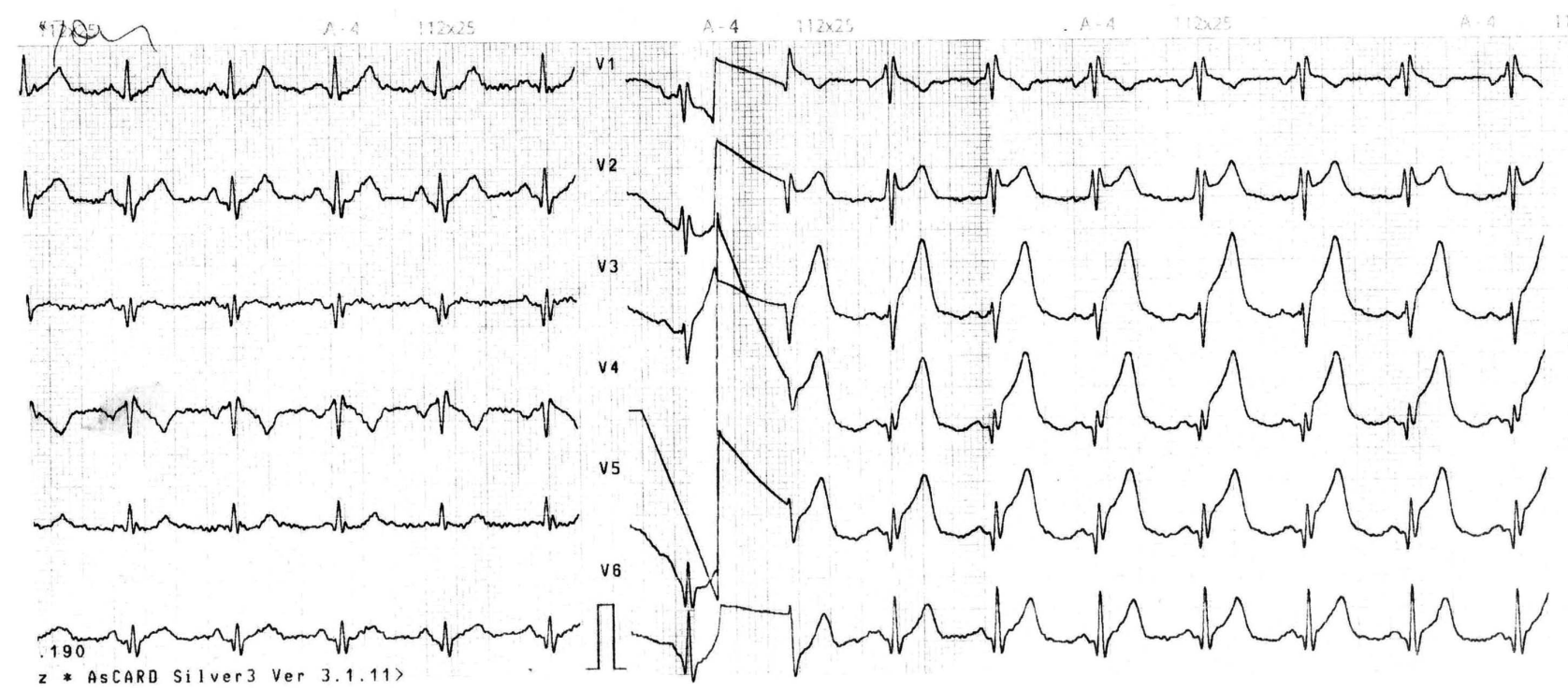

Fig. 1. First ECG transferred from ambulance to our hospital

This is an open-access article distributed under the terms of the Creative Commons Attribution-NonCommercial 4.0 International License, which permits unrestricted use, distribution, and reproduction in any medium for non-commercial purposes, provided the original author and source are credited, a link to the CC License is provided, and changes - if any - are indicated. 
Bochenek et al.

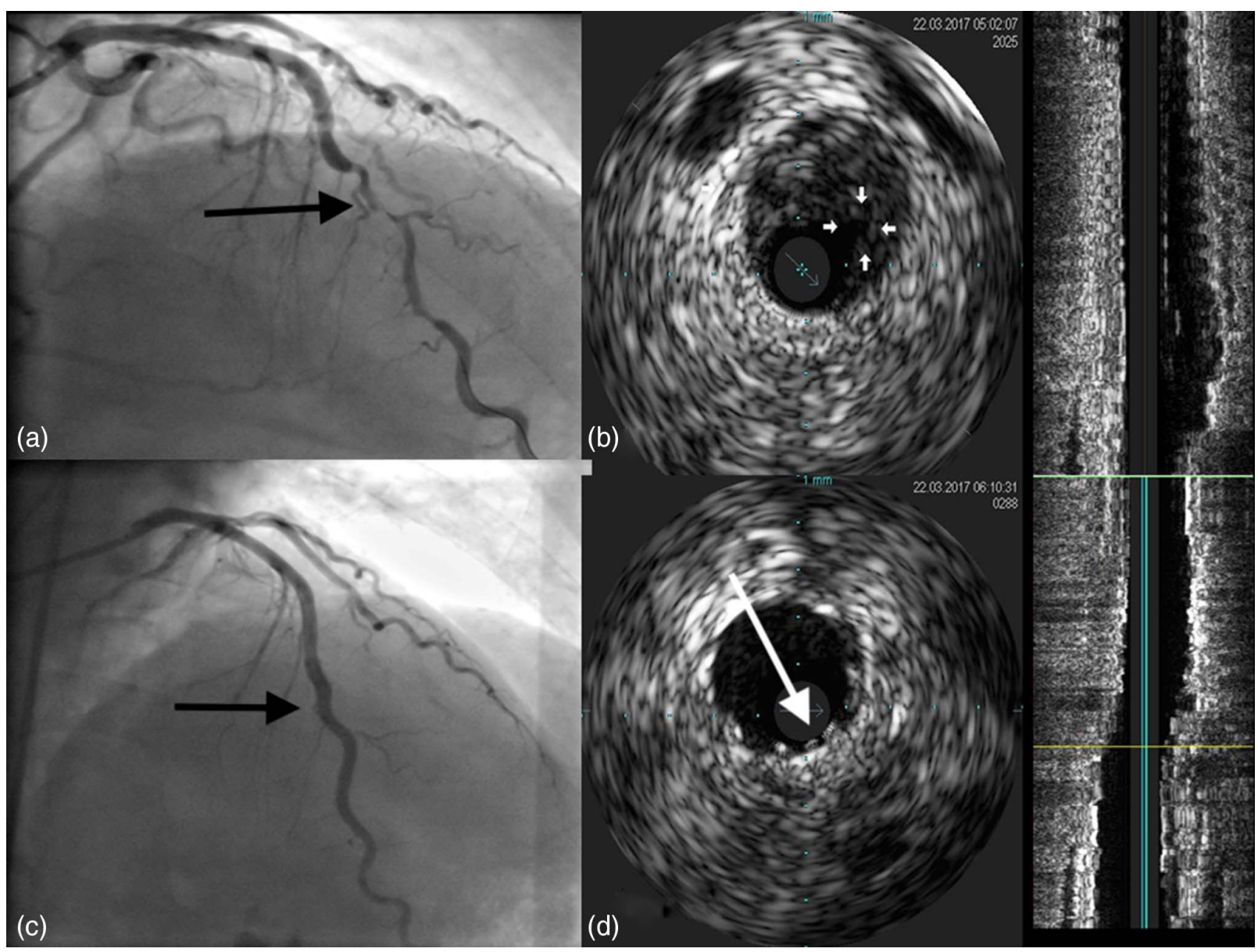

Fig. 2. Angiographic and ultrasonographic images of the artery

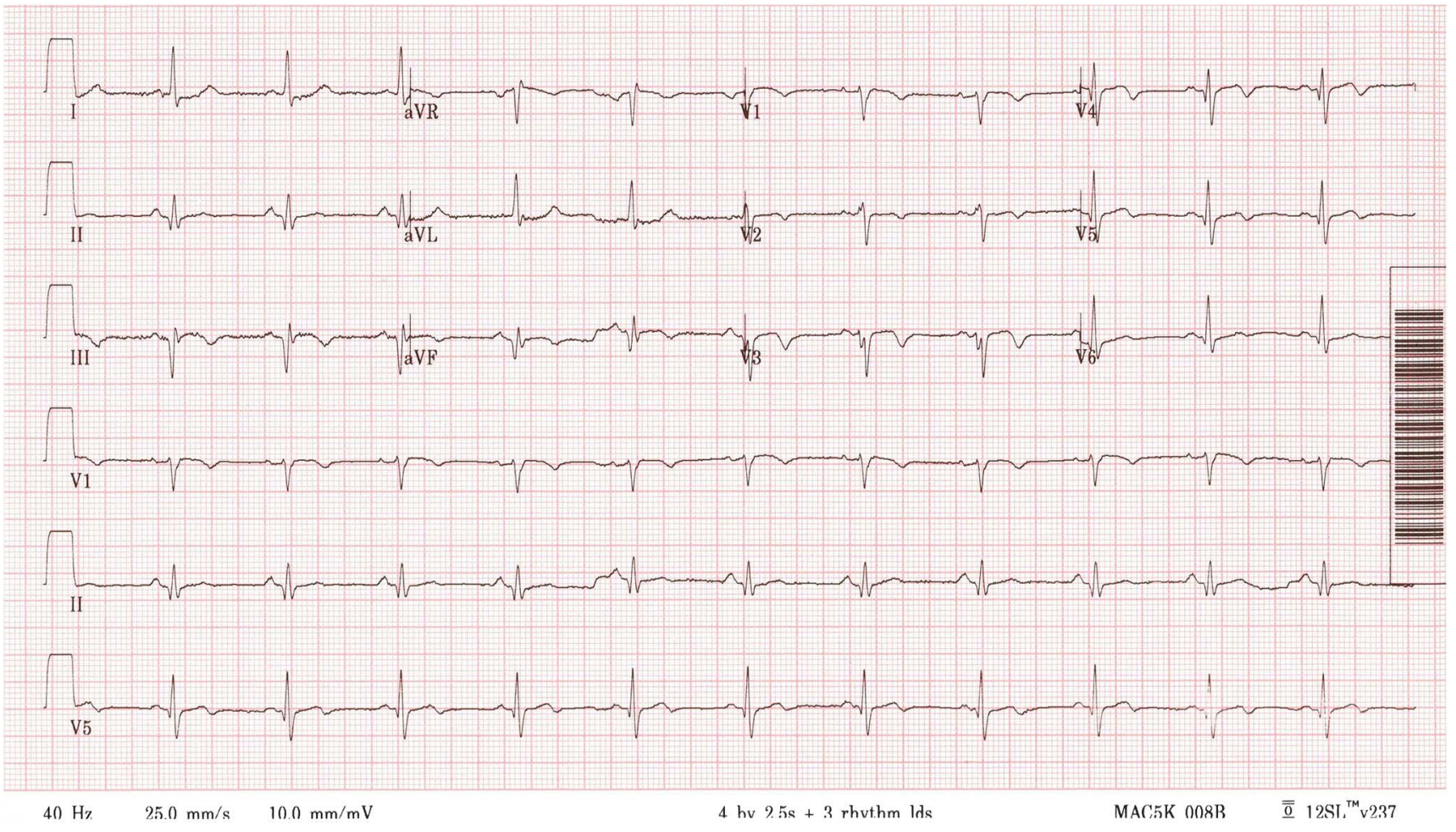

Fig. 3. Follow-up ECG of acute infarction 
A 55-year-old man without any previous cardiac history has been admitted to the Department of Cardiology, Medical University of Silesia, Poland due to anterior wall myocardial infarction (MI) [Electrocardiography (ECG); Fig. I showing typical features of anterior wall infarction ] $-9 \mathrm{~h}$ after onset of chest pain. In echocardiography, local anterior wall dysfunction has been observed, with good left ventricle ejection fraction. In angiography that was performed immediately after transfer to the hospital, long lesion in left anterior descending (LAD) coronary artery has been visualized with high angiographic suspicion of dissection and intramural coronary hematoma (Fig 2a, arrow). Right coronary artery and circumflex were free of narrowing lesions. Intravascular ultrasound (IVUS) has been performed and further confirmed the diagnosis of hematoma (Fig. $2 b$, arrows indicate dissection tear). LAD was stented using three coronary stents (Fig. 2c). IVUS has shown good position of stents (Fig $2 d$, arrow indicates stent struts). Integrilin has been used. Periprocedural time was uncomplicated. ECG after the event showed resolution of $\mathrm{MI}$ pattern and evolution of infarction has been observed (Fig. 3). Patient was discharged home in good clinical condition.

According to literature, IVUS can identify plaque rupture [1]. IVUS is an important diagnostic tool in establishing the correct diagnosis and provides a complete vessel wall assessment, which gives morphometric information on underlying hematoma [2]. To conclude, coronary dissection and coronary hematoma are the potential cause of anterior wall MI and IVUS, despite optical coherence tomography being referenced nowadays, it is still a very valuable tool in diagnosis and guiding treatment in such cases.

Funding sources: None.

Authors' contribution: None.

Conflict of interest: None.

\section{References}

1. Ge J, Chirillo F, Schwedtmann J, Görge G, Haude M, Baumgart D, Shah V, von Birgelen C, Sack S, Boudoulas H, Erbel R: Screening of ruptured plaques in patients with coronary artery disease by intravascular ultrasound. Heart 81, 621-627 (1999)

2. Antonsen L, Thayssen P, Jensen LO: Large coronary intramural hematomas: A case series and focused literature review. Cardiovasc Revasc Med 16, 116-123 (2015) 